\title{
Socio-cultural determinants of the spread of Covid 19
}

\author{
Mathew George* \\ Centre for Public Health, School of Health Systems Studies, Tata Institute of Social Sciences, Mumbai, India
}

The spread of covid 19 as well as its projected fatality is showing different pattern in different countries though the uniform response to the pandemic, namely, lockdown and physical distancing to ensure quarantine, is considered as the most appropriate option by several governments across the world. Concerns have been raised in terms of the technical competency and social feasibility countries possess in order to accomplish the goal of flattening the epidemic curve. The social, economic and ethical implications of such an intervention to the social fabric of nations are also pondered upon by social scientists. What is crucial in the spread of covid 19 is the fact that those having travel history to the infected country has a greater chance to get the infection and their primary contacts are the ones who are at immediate risk. One of the most important characteristic that explains the spread of covid cases is the 'travel behaviour' of people across nations. The current intervention of contact tracing of those individuals with travel history is premised on this assumption. The mathematical projection of cases and its spread which is the sole 'evidence' available on the potential spread usually focus only on the numerator, those cases and the ones they will immediately infect with the denominator taken in thousands or millions. In other words the susceptible population for which the projection is made is always represented as mere numbers (usually for denominator) which is assumed to possess common biological characteristics as human species and is considered as homogeneous across the globe. A population level perspective which has to be the core of any public health approach call for a more in-depth analysis of this susceptible population in terms of their social, political, economic, cultural and epidemiological characteristics. There is a need for more in-depth analysis to understand the heterogeneity of this susceptible population which might explain the dynamics of spread of epidemic in populations. There are at least three crucial socio-cultural and epidemiological characteristics that need to be focused upon in the context of covid 19 .

First and foremost is the proportion of population that potentially engage in international travel across nations in a given time period. For instance going by the immigration data, the proportion of immigrant population to the total population for countries in the Europe and US are much higher than it is in the Eastern countries like China, Japan, Korea and India. This is in the range of 9-17\% for Europe whereas for Eastern and Asian countries, it hovers in the range of 0.4 to $3 \%$. Adding on to this is the purpose of international travel across nations whether it is part of trade, employment, tourism and sporting activities, the latter two is high in the western population, especially for Italy and Spain. Thus it is obvious that at any given point of time the proportion of population engaged in international travel is much higher in the European, US and other western countries where the pace of spread was faster than in Asian, African and middle eastern region. There seems to be an association which one can attribute to the pace of spread of covid 19 in a country and the proportionate population with international travel history.
Second important characteristic of covid 19 is the possibility of close contact across population with those infected owing to the mode of spread of the disease as close contact with a case is identified as the mode of transmission. This is where the culture of the susceptible population become evident and their behavior need detailed scrutiny. For instance, Italy was attributed to have a culture of close interaction (physical intimacy) as compared to Japan (culturally maintaining greater physical distance). This human behavior of maintaining or not maintaining physical distance is an outcome of a nation's culture which has deeper roots in their national heritage and tradition. In LMICs where there is poor living and working conditions the very possibility of maintaining physical distance even during pandemics like Covid is impossible. Any effort to suddenly change the behavior to avoid spread is not feasible and will face resistance from the people as demonstrated in several countries. This further gets complicated when there is a failure to understand that physical distancing in a given social context is unaffordable for a huge population by virtue of their engagements as part of their daily living and working conditions. Any attempt to trivialize human behavior change as that which can be accomplished by manipulating individual consciousness without changing the social context has always failed miserably in Public health. Failure to follow suit by people should not result in 'victim blaming' and any attempt to introduce punitive acts through legal means will have serious ethical implications as it is the most deprived and vulnerable who will be at the receiving end.

Third important factor of a population characteristic that is crucial in any epidemic spread is the level of 'natural immunity' among susceptible population. It is a well-known fact that immunity status of a population decides the pace at which epidemics spread or not. In the current situation, it is important to acknowledge how countries like India in terms of its exposure to infections at the population level interact with the occurrence of covid 19. This will be the key to decide the extent of community spread in low and middle income countries. Will the spread of epidemic changes when the susceptible population has a history of exposure to a range of epidemics like influenza, malaria, dengue, TB and pneumonia? If so, will it be similar to other South Asian population with similar disease history. Recent epidemiological study identifies correlation between universal BCG vaccination policy of nations and reduction in morbidity and mortality for covid 19 . What are the critical population characteristics of African nationals that will interfere with covid spread having exposed to the recent Ebola outbreak in the region? There is a clear paucity of evidence in this regard. It is necessary to consider the heterogeneity of the susceptible population in terms of their social, political, economic, cultural and epidemiological

${ }^{\star}$ Correspondence to: Mathew George, Professor, Centre for Public Health, School of Health Systems Studies, Tata Institute of Social Sciences, Mumbai, India, Tel +22 25525512/+91 9969607444; E-mail: mathewg@tiss.edu

Received: April 13, 2020; Accepted: April 21, 2020; Published: April 24, 2020 
characteristics while interpreting the spread of epidemics. This calls for more population level interdisciplinary studies to understand the dynamics of disease transmission within the family, workplace and community settings across nations. This is all the more relevant during epidemics as it deals with life and death decisions of people [1-7].

\section{Disclaimer}

Views expressed in the article are personal.

\section{References}

1. Singh R, Adhikari R (2020) Age-structured impact of social distancing on the COVID-19 epidemic in India. Quantitative Biology.

2. http://theconversation.com/which-countries-have-the-most-immigrants-113074
3. Sander N, Abel GJ, Riosmena F (2013) The future of international migration: Developing expert-based assumptions for global population projections. Vienna Institute of Demography Working Papers, ECONSTOR.

4. Anderson RM, Heesterbeek H, Klinkenberg D, Hollingsworth TD (2020) How will country-based mitigation measures influence the course of the COVID-19 epidemic? The Lancet 395: 931-934.

5. https://www.liverpoolecho.co.uk/news/uk-world-news/six-reasons-coronavirusspread-fast-17901773

6. https://www.businessinsider.in/science/news/japan-only-has-924-coronavirus-casesthis-may-be-from-a-lack-of-testing-and-there-are-more-cases-of-infection-thanthought-/articleshow/74720252.cms

7. Miller A, Reandelar MJ, Fasciglione K, Roumenova V, Li Y, et al. (2020) Correlation between universal BCG vaccination policy and reduced morbidity and mortality for COVID-19: an epidemiological study. medRxiv.

Copyright: $\odot 2020$ George M. This is an open-access article distributed under the terms of the Creative Commons Attribution License, which permits unrestricted use, distribution, and reproduction in any medium, provided the original author and source are credited. 